\title{
INFLUENCIA DE LA NO LINEALIDAD DEL MATERIAL EN LA DINÁMICA DE ESTRUCTURAS ARTICULADAS DE MADERA
}

\author{
(INFLUENCE OF MATERIAL NON LINEARITY IN THE ARTICULATED WOOD STRUCTURES \\ DYNAMICS)
}

Enrique López Hernández, Dr. Ciencias Físicas y Manuel Muñoz Vidal, Arquitecto

Dpto. Tecnología de la Construcción. Univ. de La Coruña

ESPAÑA

Fecha de recepción. 27-VI-96 $660-6$

\section{RESUMEN}

Se estudia el efecto del comportamiento no lineal del material en el movimiento de una estructura de barras de madera articuladas, mediante un método de cálculo itexativo con masas concentradas en los nodos.

\section{SUMMARY}

This paper studies the effect of the non-linear behavior of the material in the movement of an articulate structure made of wooden bars, by means of an iterative of calculation method with lumped masses in the nodes

\section{Introducción}

El concepto de linealidad es una idealización acerca del comportamiento real de los materiales que forman las estructuras. Esta aproximación se utiliza ampliamente en el análisis estructural, debido a que, generalmente, da resultados suficientemente correctos y a que simplifica en gran manera los cálculos a realizar.

Esto es especialmente cierto en el caso de análisis dinámico de estructuras donde, gracias a las pequeñas amplitudes usuales en este tipo de movimiento, el comportamiento lineal o cuasi lineal se da generalmente por supuesto [1].

Esto conduce a que los métodos de cálculo habituales se basen en el análisis del movimiento en el dominio de las frecuencias, considerando entonces el movimiento de las estructuras como suma o superposición lineal de movimientos más sencillos o vectores propios, cada uno de los cuales está asociado a una frecuencia de vibración característica. En el caso de comportamiento perfectamente lineal, los vectores propios son los conocidos como modos normales de vibración, siendo varios los enfoques para el caso cuasi lineal; es decir, cuando los términos no lineales son pequeños en comparación con los términos lineales, pero distintos de cero. Nacen así, por ejemplo, distintas aproximaciones, como los vectores de Ritz, utilizados en códigos ampliamente difundidos tales como el SAP90 [2].

En este artículo se propone realizar una evaluación de los efectos no lineales del material en la dinámica de una estructura formada por barras de madera unidas en sus extremos mediante articulaciones (Fig. 1). Estudiamos el caso particular de la madera por ser un material en el cual el comportamiento a tracción suele diferir notablemente del comportamiento a compresión y, por lo tanto, un cambio de signo en las tensiones de las barras debido a cargas variables con el tiempo se traducirá en un comportamiento no lineal de la estructura. Todo ello englobado en el marco de un método iterativo propio, que permite el análisis en el tiempo de la dinámica de una estructura articulada fuertemente no lineal. 


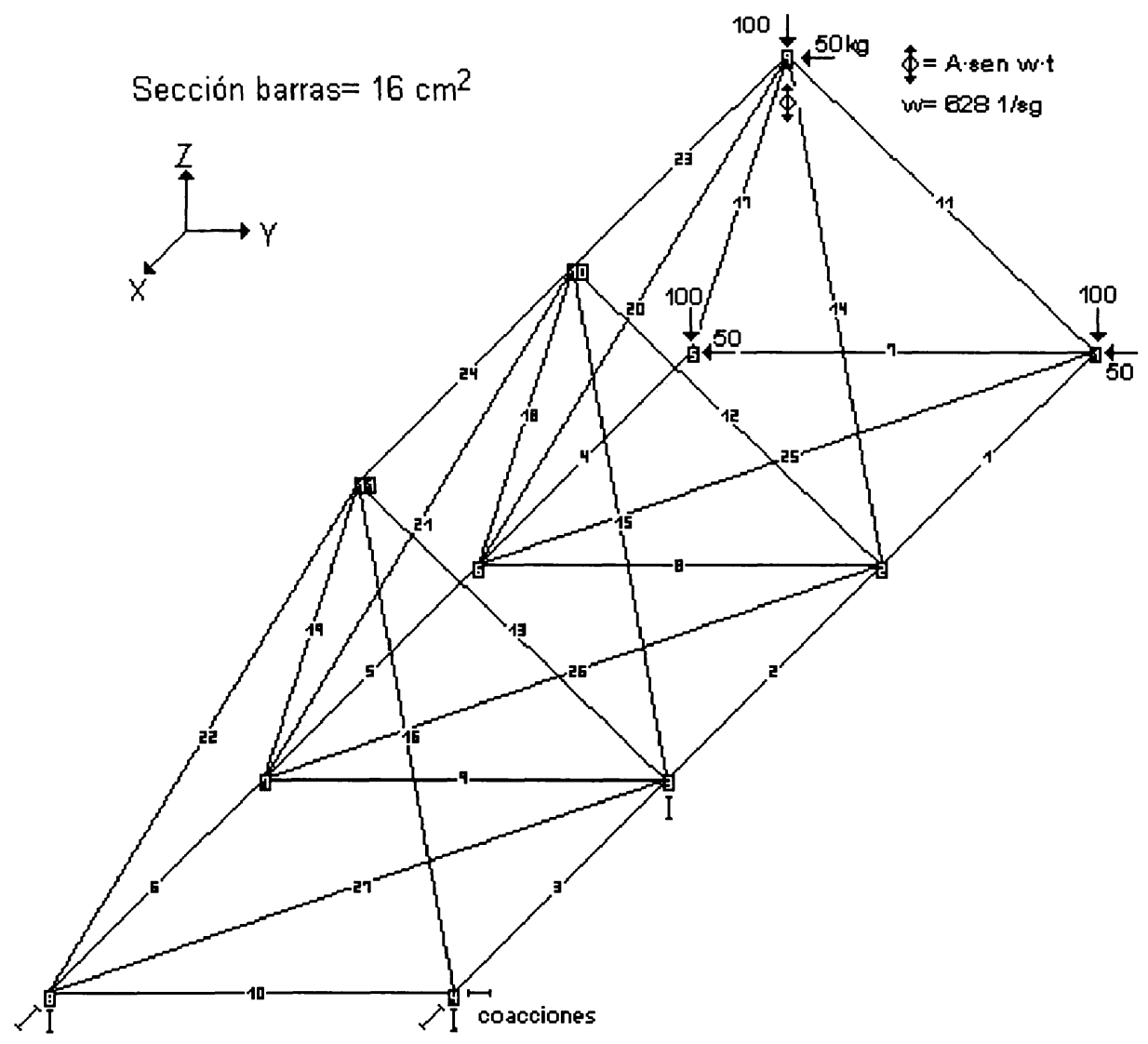

Fig. 1

\section{Definición del problema}

Consideremos una estructura formada por barras de madera unidas entre sí mediante nudos articulados y sujeta a unas condiciones de contorno o coacciones y a unas fuerzas exteriores dadas (ver Fig.1). Como es habitual, la primera aproximación consiste en discretizar la geometría de la misma mediante el método de elementos finitos. En efecto, si consideramos que el comportamiento de las barras se puede describir por las fuerzas en los extremos de las mismas, se puede demostrar que sus ecuaciones de movimiento se pueden expresar en la forma [3]:

$$
\mathbf{K} \cdot \mathbf{U}+\mathbf{C}+\mathbf{M} \cdot \ddot{\mathbf{U}}=\mathbf{R}(\mathbf{t})
$$

donde:

$\mathbf{K}$ - matriz de rigidez

U - matriz de desplazamientos

C - matriz de amortiguamiento

M - matriz de masas consistente

$\ddot{\mathbf{U}}$ - matriz de aceleraciones

$\mathbf{R}$ - matriz de fuerzas exteriores

En el caso más sencillo de una sola barra de material lineal y expresando la ecuación (1) en coordenadas locales resulta (Fig. 2) [4]

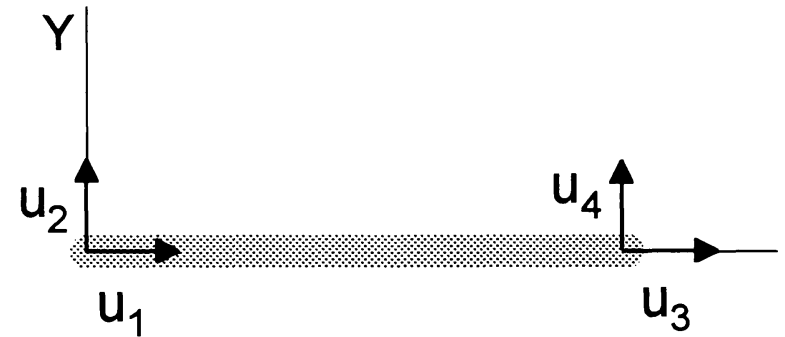

Fig. 2

$$
U=\left(\begin{array}{l}
u_{1} \\
u_{2} \\
u_{3} \\
u_{4}
\end{array}\right) \quad \ddot{U}=\left(\begin{array}{c}
\ddot{u}_{1} \\
\ddot{u}_{2} \\
\ddot{u}_{3} \\
\ddot{u}_{4}
\end{array}\right)
$$

donde:

$\rho$ - densidad

L - longitud 
A - sección transversal

E - módulo de elasticidad longitudinal de la barra

Dependiendo del tipo de amortiguamiento la matriz $\mathbf{C}$ adoptará una forma $u$ otra. $\mathbf{M}$ es la denominada matriz de masa consistente, porque aparece de forma natural al establecer las ecuaciones de movimiento en el método de elemento finitos. Para simplificar el cálculo reducimos esta matriz no diagonal mediante la aproximación de masas concentradas [5], suponiendo que las barras se pueden representar por dos masas situadas en ambos extremos de lasbarras y despreciando por tanto el acoplamiento dinámico existente entre los extremos de las barras. La matriz de masas concentradas resulta ahora diagonal, quedando para el caso de una barra simple en la forma:

$$
M=\frac{\rho \cdot L \cdot A}{2} \cdot\left(\begin{array}{llll}
1 & 0 & 0 & 0 \\
0 & 1 & 0 & 0 \\
0 & 0 & 1 & 0 \\
0 & 0 & 0 & 1
\end{array}\right)
$$

donde los efectos de inercia asociados con los grados de libertad rotacionales se han supuesto también despreciables. De esta manera se reduce la ecuación de movimientode una barra a dos ecuaciones de movimiento de nodos.

Ampliando esta formulación a la estructura de la Fig. 1, las matrices de movimiento se podrán descomponer en bloques a partir de las matrices (2), teniendo en cuenta la orientación espacial de las barras y que, en el caso de nudos donde searticulan varias barras, la masade cadauno corresponderá a la semisuma de las masas de la barras que llegan a él.

En general la no linealidad en una estructura surge, bien en la matriz de amortiguamiento $\mathbf{C}$, bien en el material a través de la magnitud $\mathrm{E}$ o bien en las condiciones de contorno. En nuestro caso la no linealidad en la ecuación (1) la vamos a estudiar cuando proviene, no de la matriz C, que consideraremos nula, sino de la matriz K. En efecto, como es sabido, muchos son los materiales de construcción que exhiben propiedades elásticas no proporcionales, de tal manera que la curva tensión-deformación no es una recta , sino una función más o menos complicada. Una curva de tensión-deformación típica de la madera, cuando los esfuerzos son en la dirección de las fibras, puede ser como la indicada en la Fig. 3 [6]:

Aproximando esta curva la podemos reducir a dos rectas, de manera que obtenemos un tipo de no linealidad especialmente significativo, que es aquél en el que el material a tracción se comporta diferente de a compresión, es decir, las deformaciones positivas tienen un módulo de elasticidad $\mathrm{E}^{+} \mathrm{y}$ otro distinto las negativas $\mathrm{E}^{-}$. La gráfica de trabajo del material se podrá aproximar entonces a la forma indicada en la Fig. 4.

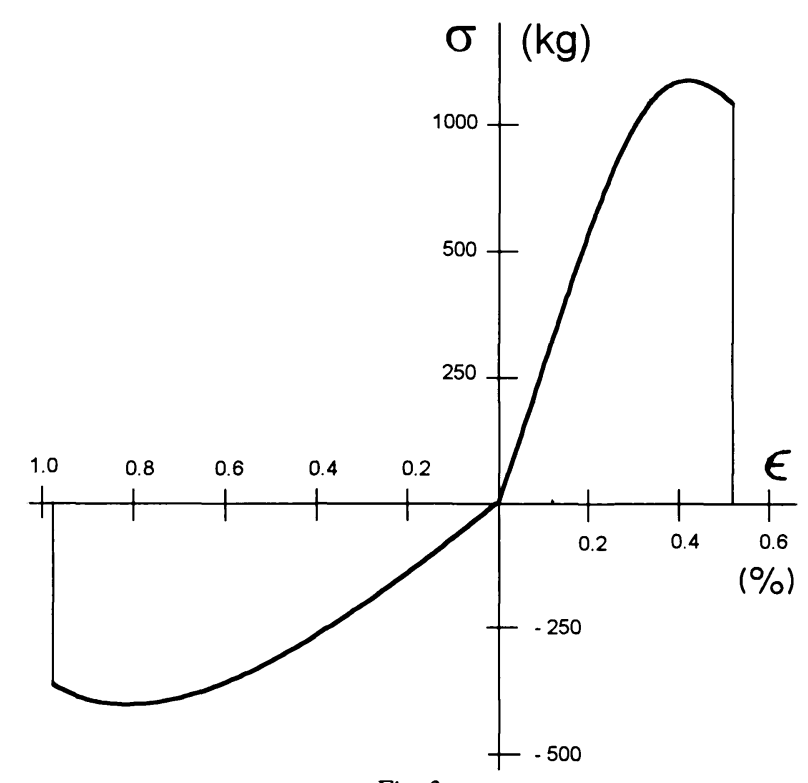

Fig. 3

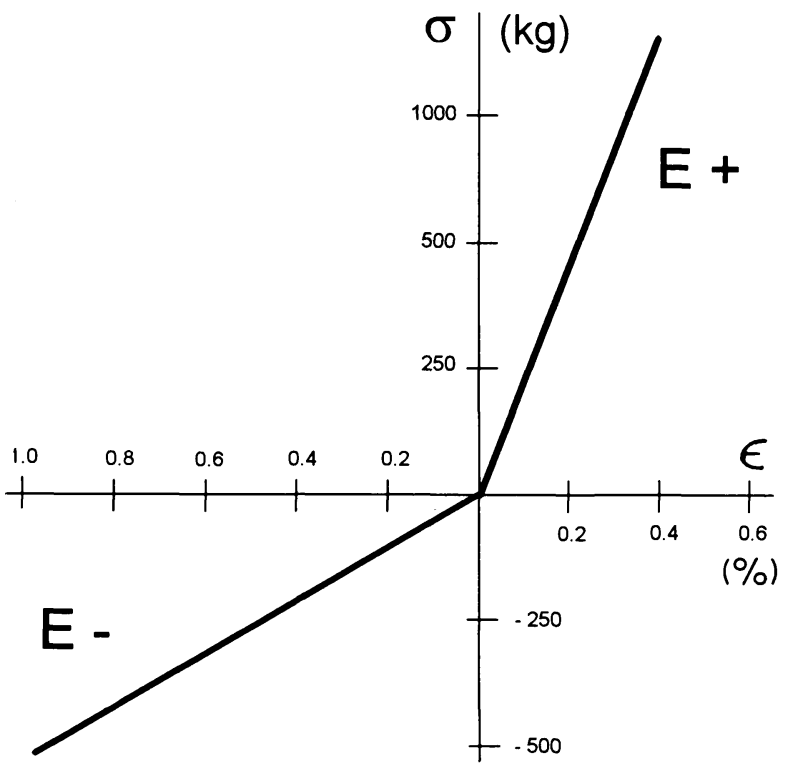

Fig. 4

Está claro que con un material de esta clase los fenómenos no lineales aparecerán cuando al menos una barra pase de trabajar atracción atrabajar a compresión oviceversa. Para estos materiales, y en el caso sencillo de la Fig. 2, la matriz de rigidez $\mathbf{K}$ se puede expresar en la forma:

$$
K=\frac{\hat{E} \cdot A}{L} \cdot\left(\begin{array}{cccc}
1 & 0 & -1 & 0 \\
0 & 0 & 0 & 0 \\
-1 & 0 & 1 & 0 \\
0 & 0 & 0 & 0
\end{array}\right)
$$




$$
\hat{E}=\left[\frac{\left(u_{1}-u_{3}\right)}{\left|u_{1}-u_{3}\right|}\right] \cdot \frac{\left(E^{+}-E^{-}\right)}{2}+\left(\frac{E^{+}+E^{-}}{2}\right)
$$

En la estructura de madera analizada (Fig. 1), se consideró $\mathrm{E}^{+}=220 \cdot 10^{3} \mathrm{~kg} / \mathrm{cm}^{2}$ y $\mathrm{E}^{-}=60 \cdot 10^{3} \mathrm{~kg} / \mathrm{cm}^{2}$.

Las fuerzas se consideran actuando únicamente sobre los nudos y se dividen en estáticas o constantes y dinámicas o variables en el tiempo. Se analizaron dos series de movimientos de la estructura que se diferencian en las fuerzas estáticas. En un caso son únicamente el peso propio de la estructura (caso B) y en el otro se añadieron las fuerzas que se indican en la Fig. 1 (caso A). En ambos casos se mantiene una carga dinámica senoidal actuando en el nudo 9 , con dirección vertical.

En cualquier caso se analiza el movimiento de la estructura debido a la fuerza dinámica a partir del equilibrio y deformación de la estructura debida a las fuerzas estáticas. En la Tabla 1 se indican las tensiones de equilibrio de las barras en ese momento para cada caso.

TABLA 1

\begin{tabular}{|c|c|c|}
\hline$n^{0}$ barra & $\begin{array}{c}\text { Caso A } \\
\text { axil }(\mathrm{kg})\end{array}$ & $\begin{array}{l}\text { Caso B } \\
\text { axil (kg) }\end{array}$ \\
\hline 1 & 175 & -0.7 \\
\hline 2 & 401 & -5.2 \\
\hline 3 & 625 & -14.3 \\
\hline 4 & -25 & -0.7 \\
\hline 5 & -400 & -5.2 \\
\hline 6 & -775 & -14.3 \\
\hline 7 & 75 & -0.7 \\
\hline 8 & 200 & -1.2 \\
\hline 9 & 199 & -1.2 \\
\hline 10 & 75 & -14.3 \\
\hline 11 & 61 & 2.5 \\
\hline 12 & -31 & 7.8 \\
\hline 13 & -550 & -36.7 \\
\hline 14 & 31 & -4.1 \\
\hline 15 & 31 & -9.6 \\
\hline 16 & 550 & 35.1 \\
\hline 17 & 61 & 1.7 \\
\hline 18 & 214 & 7.0 \\
\hline 19 & 214 & 12.6 \\
\hline 20 & -214 & -3.3 \\
\hline 21 & -214 & -8.9 \\
\hline 22 & -215 & -14.2 \\
\hline 23 & 125 & 4.8 \\
\hline 24 & 274 & 18.4 \\
\hline 25 & -283 & -0.4 \\
\hline 26 & -283 & -0.4 \\
\hline 27 & 18 & 28.4 \\
\hline
\end{tabular}

\section{Método de cálculo}

Para resolver la ecuación (1) aprovechamos, en primer lugar, el hecho de que $\mathbf{M}$ es diagonal con determinante distinto de cero de manera que se puede despejar la matriz de aceleración y expresarla en la forma:

$$
\ddot{\mathbf{U}}=\mathbf{M}^{-1} \cdot[\mathbf{R}-\mathbf{C}-\mathbf{K} \cdot \mathbf{U}]
$$

Para no estar condicionados por el comportamiento más o menos lineal de la estructura, la ecuación (7) se resuelve mediante un método iterativo con un esquema de integración en el tiempo de tipo implícito [7]. En efecto, dadas las posiciones $U_{t}$ y las velocidades $\dot{U}_{t}$ de los nudos en un instante $\mathbf{t}$, las posiciones y velocidades en un instante $\mathbf{t}+\Delta \mathbf{t}$ se calculan según los pasos siguientes:

$1^{\circ}$ Cálculo de las posiciones $U_{p}$, velocidades $U_{p}$ provisionales en un instante $t+\Delta t$ suponiendo que la aceleración de los nudos durante el intervalo $\Delta \mathrm{t}$ se mantiene constante e igual a la aceleración en el instante $t$.

$$
\begin{aligned}
& \dot{U}_{p}^{t+\Delta t}=\dot{U}^{t}+\ddot{U}^{t} \cdot \Delta t \\
& U_{p}^{t+\Delta t}=U^{t}+\left(\dot{U}^{t}+\dot{U}_{p}^{t+\Delta t}\right) \cdot \frac{\Delta t}{2}
\end{aligned}
$$

$2^{\circ}$ Cálculo de las posiciones y velocidades finales en el instante $\mathrm{t}+\Delta \mathrm{t}$ suponiendo las aceleraciones uniformemente variables entre el valor en el instante $t \mathrm{y}$ el valor provisional en el instante $t+\Delta t$ deducido de la ecuación (7) para las posiciones y velocidades provisionales (8) y (9).

$$
\begin{aligned}
& \ddot{U}_{p}^{t+\Delta t}=M^{-1} \cdot\left[R-C-K \cdot U_{p}^{t+\Delta t}\right] \\
& \dot{U}^{t+\Delta t}=\dot{U}^{t}+\left(\ddot{U}^{t}+\ddot{U}_{p}^{t+\Delta t}\right) \cdot \frac{\Delta t}{2} \\
& U^{t+\Delta t}=U^{t}+\left(2 \cdot \dot{U}^{t}+\dot{U}^{t+\Delta t}+\ddot{U}^{t} \cdot \frac{\Delta t}{2}\right) \cdot \frac{\Delta t}{3}
\end{aligned}
$$

En el diagrama adjunto (Fig. 5) se representa el esquema de integración elegido

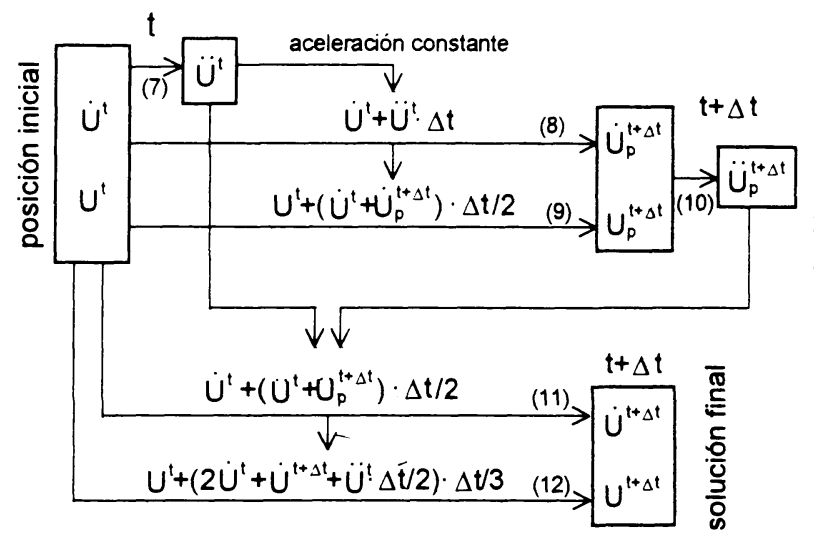

aceleración uniformemente variable

Fig. 5

La elección de intervalo de tiempo de integración $\Delta \mathrm{t}$ es importante en todo este tipo de esquemas de integración, necesitándose que sea lo suficientemente pequeño para que la solución converja a valores significativos y para que el comportamiento iterativo sea correcto; en nuestro caso $\Delta t=1 \cdot 10^{-4} \mathrm{seg}$.

Estas ecuaciones se resuelven para unas $3 \cdot 10^{3}$ iteraciones sucesivas, de tal manera que se analizan $0,3 \mathrm{seg}$. de 
movimiento con una precisión en las frecuencias de unos $20 \mathrm{seg}^{-1}$ aproximadamente.

A las aceleraciones obtenidas $\ddot{U}(t)$ en nudos escogidos, se les aplica el algoritmo de la transformada rápida de Fourier (FFT) para estudiar las características del movimiento en el dominio de las frecuencias, obteniendo las correspondientes distribuciones de densidad de potencia.

\section{Resultados}

Como se indicó en el apartado 2, se analizaron dos series de movimientos A y B. En las Figs. 6 y 7 se presentan los espectros del nudo 1 en las direcciones $Y$ y $Z$ cuando están actuando, aparte del peso propio, las cargas estáticas y dinámicas externas (caso $\mathrm{A}$ ).

Los gráficos sucesivos de cada caso se diferencian en la amplitud de la carga dinámica, que toma los sucesivos valores: $5,10,50,100,200,500 \mathrm{~kg}$, mientras que su frecuencia permanece constante en $\omega=628$.

En las Figs. 8 y 9 se presentan los espectros del nudo 1 en las direcciones $\mathrm{Y}$ y $\mathrm{Z}$ cuando están actuando solamente el peso propio y las cargas dinámicas (caso B).

\section{FFT dirección $Y$}
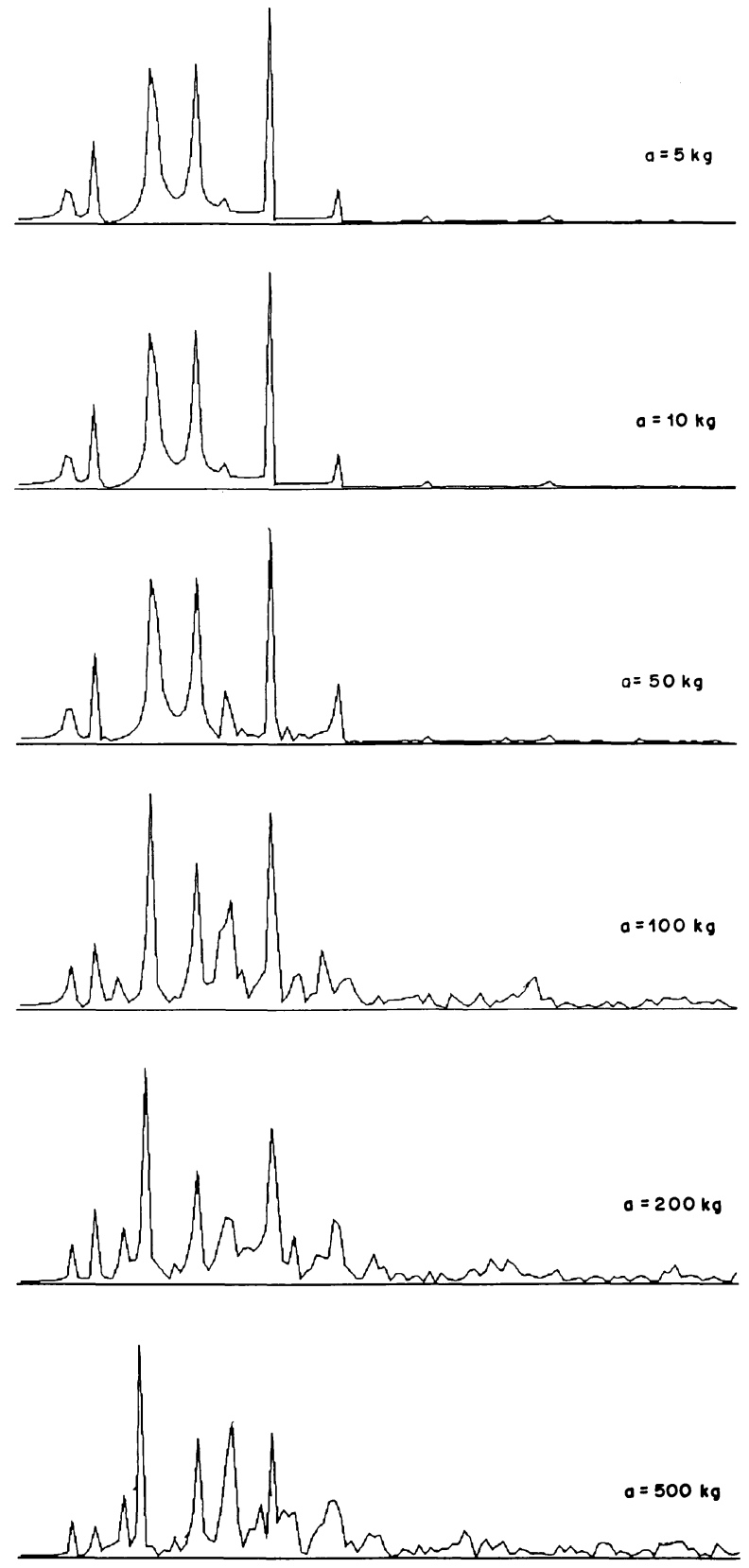

Fig. 6

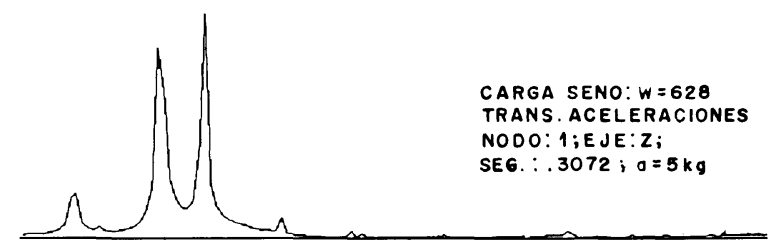

0

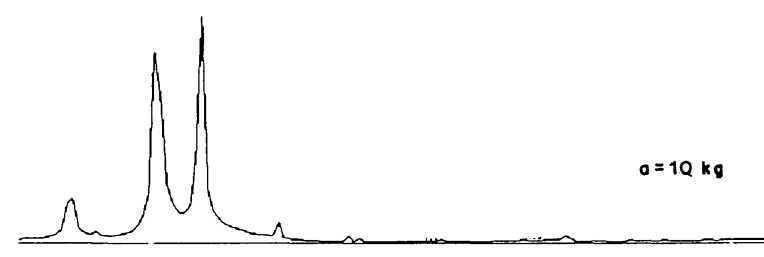

12,27

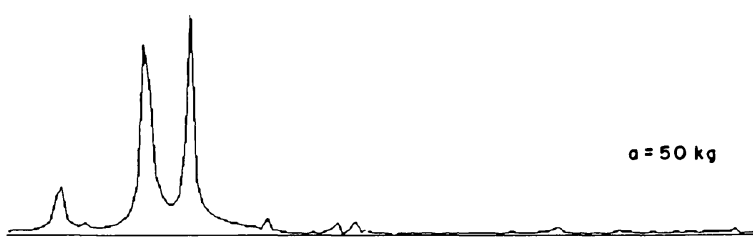

4,12 ,

14,15

27

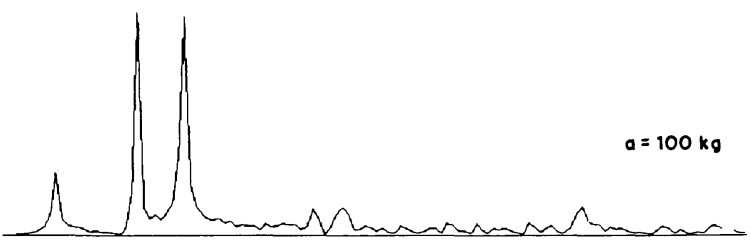

4,11 ,

12,14

15,17 ,

23, 27

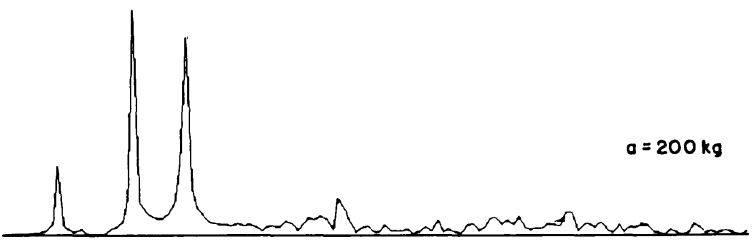

$1,4,7$,

10,11 ,

12,14 ,

15,17 ,

18,19 ,

20,23 ,

24,27

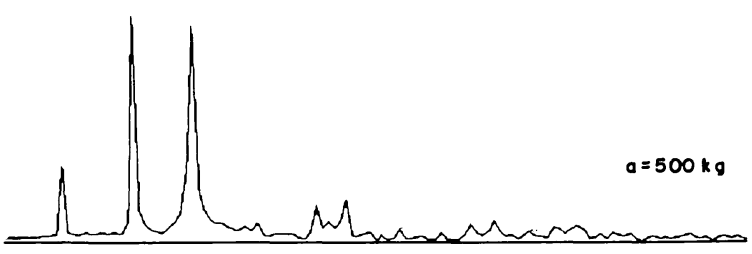

Fig. 7 
FFT dirección $Y$
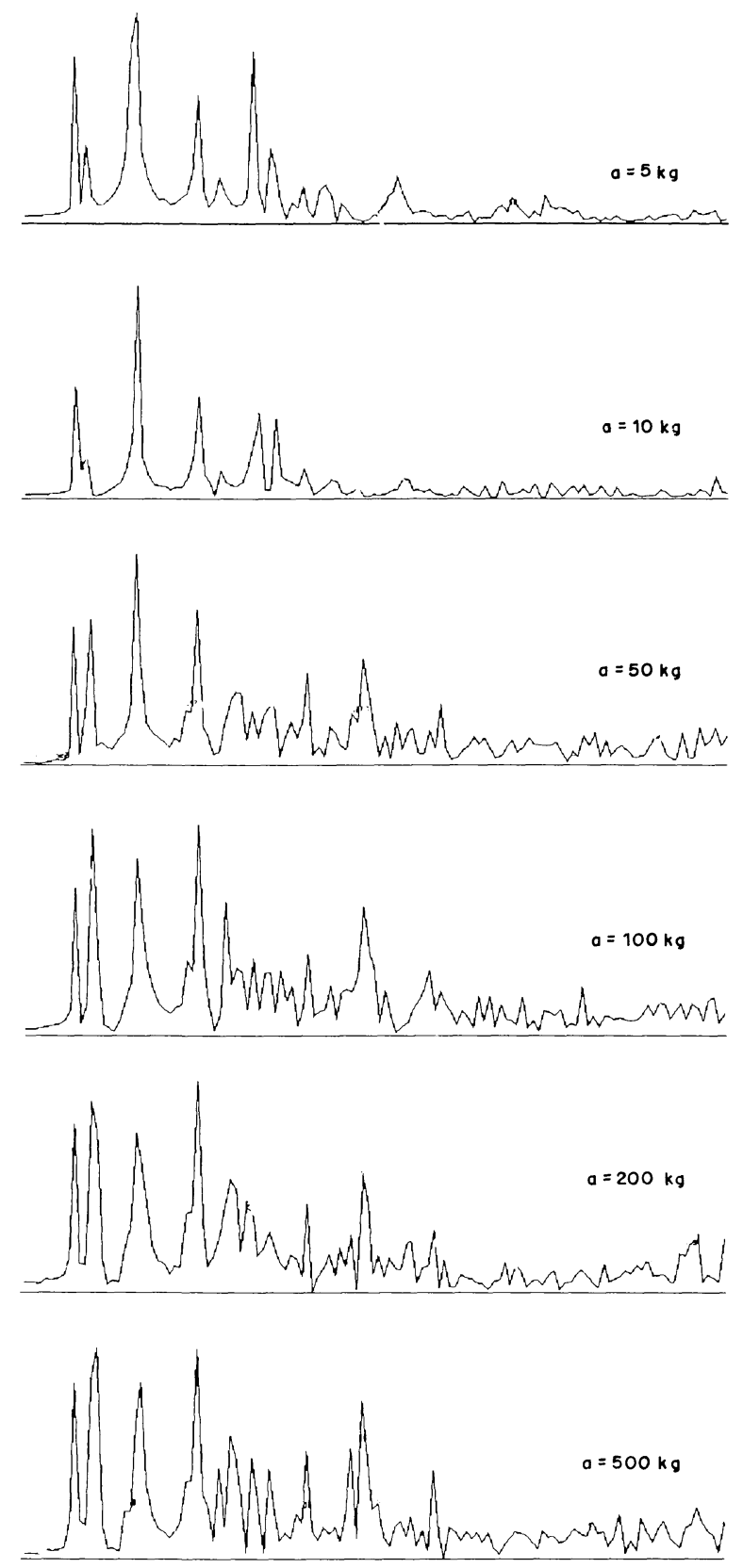

Fig. 8

\section{Comentarios y conclusiones}

Se han estudiado los efectos no lineales asociados al material, en una estructura articulada de madera. Con tal fin, se desarrolló un método de cálculo iterativo utilizando un modelo de masas concentradas en los nudos y se aproximó el comportamiento no lineal del material suponiendo que el módulo de elasticidad a tracción $\mathrm{E}^{+}$y el módulo a compresión $\mathrm{E}^{-}$son constantes, aunque distintos. Se la ha sometido a fuerzas constantes y variables en el tiempo, modificando las magnitudes relativas de las mismas con el fin de determinar cómo y cuándo se pone de manifiesio ei comportamiento no lineal

\section{$1,4,7$ \\ 8,9 \\ 25,26}

$$
\begin{aligned}
& 1,2,4 \\
& 7,8,9 \\
& 11,14 \\
& 17,20 \\
& 25,26
\end{aligned}
$$

Todas

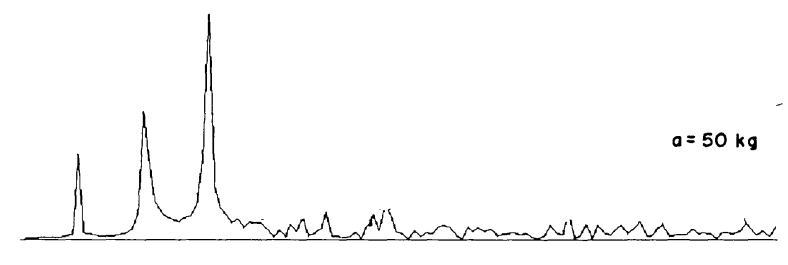

Todas

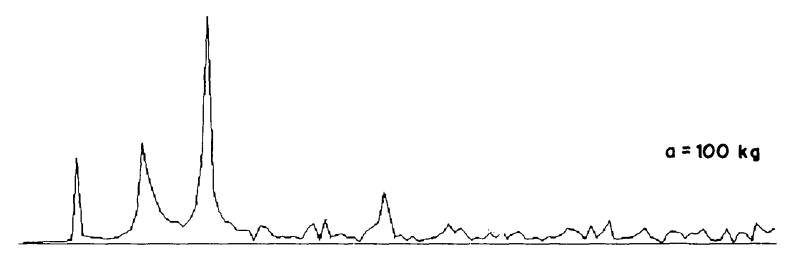

Todas

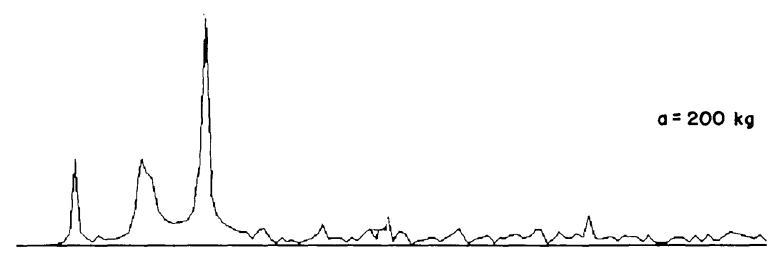

Todas

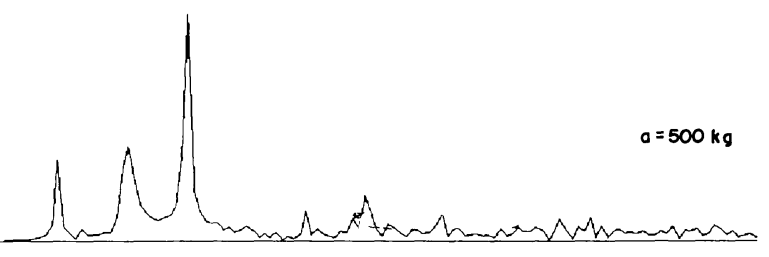

Fig. 9

Según los resultados obtenidos, para pequeñas fuerzas dinámicas frente a las cargas estáticas y las tensiones de las barras, la estructura se comporta de manera lineal y no varía la distribución de densidad de potencia (Fig.6) al variar la amplitud de la fuerzadinámica. Esto se corresponde con que ninguna barra cambia su modo de trabajo de tracción a compresión o viceversa.

A medida que las cargas dinámicas crecen, las barras pueden cambiar de estado mecánico (trabajan en las ramas de compresión y tracción) y, consecuentemente, los fenómenos no lineales.aumentan, apareciendo nuevos picos en las distribuciones (Figs. 6 y 7) y alterándose la 
frecuencia de los picos existentes. Las distribuciones de densidad dependen ahora de la amplitud de la carga dinámica.

Todos estos resultados conducen a que, en el estudio de la dinámica de una estructura articulada de madera, son importantes los fenómenos no lineales, si las cargas dinámicas son del orden de las cargas estáticas o de las tensiones de equilibrio de las barras.

\section{BIBLIOGRAFIAA}

[1] S. H. CRANDALL; Nonlinearities in structural dynamics. The Shock and Vibration Digest. Vol. 6, nº 8. August 1974. pp. $2-14$
[2] E. L. WILSON, A. HABIBULLAH; SAP 90. Structural Analysis Users Manual. CSI. Berkeley, California, USA.

[3] S. S. RAO; Mechanical Vibrations. $3^{\text {a }}$ Ed. Addison-Wesley Co. Reading, Massachusetts. 1995. Chap. 21-2.

[4] M. L. JAMES, G. M. SMITH, J. C. WOLFORD, P. W. WHALEY; Vibration of Mechanical and Structural Systems. Harper\&Row Publishers, New York 1989. Chap. 6-7.

[5]R. ARGÜELLES ÁLVAREZ; Cálculode Estructuras. E.T.S.I. de Montes, Madrid 1981. Cap. XXI.

[6] R. ARGÜELLES ÁLVAREZy F. ARRIAGA MARTITEGUI; Diseño y cálculo de estructuras de madera, C.O.A.M.

[7] KLAUS-JÜRGEN BATHE; Finite Element Procedures in Engineering Analysis. Sec.9 Prentice-Hall. Inc. New Jersey.

\section{Publicaciones del IETCC/CSIC}

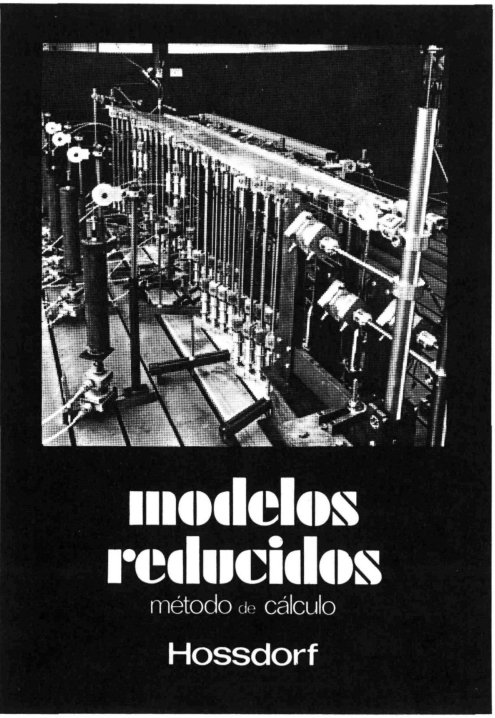

Modelos reducidos. Método de cálculo

H. Hossdorf, Ingeniero Civi

La técnica de los ensayos en modelos reducidos de estructuras sufre hoy dia una decisiva metamorfosis. Hasta hace poco era un medio más bien de artesania, que no siempre era tomado en serio por los académicos teorizantes para comprender el comportamiento resistente de las estructuras complejas $y$ al que se acudió las más de las veces, como a un último remedio debido a sus indiscutibles insuficien. cias. Sin embargo, en poco tiempo y gracias a su conexión con los ordenadores digitales, se ha trans. formado en un instrumento científicamente valioso, que no puede quedar a un lado en la práctica diaria del Ingeniero Proyectista.

Un volumen encuadernado en cartoné plastificado con lomo de tela, de $17 \times 24 \mathrm{~cm}$, compuesto de 250 páginas, 158 figuras y fotografias.

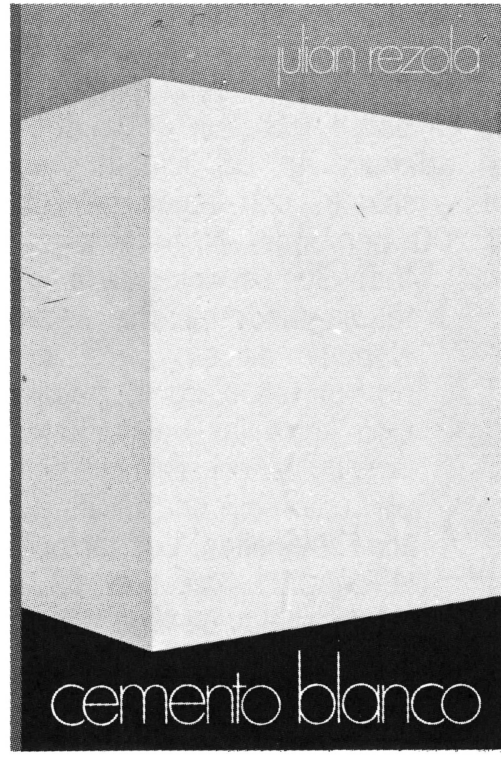

Cemento blanco

Julián Rezola

Ingeniero Quimico Dipl. I. Q. S

Sabido es que existe una extensa y documentada bibliografia sobre el cemento gris: en cambio, no puede decirse lo mismo acerca del cemento portland blanco, ya que los escritos existentes se refieren tan sólo a algunas peculiaridades que le distinguen de aquél.

El autor nos ofrece sus profundos conocimientos y su larga experiencia tanto en laboratorio como

La parte descriptiva del libro se complementa con gráficos, diagramas y fotografias de gran utilidad, destinados a conseguir la aplicación apropiada de este aglomerante.

Un volumen encuadernado en cartoné policerado, de $17,4 \times 24,3 \mathrm{~cm}$, compuesto de 395 páginas, numerosas figuras, tablas y ábacos.

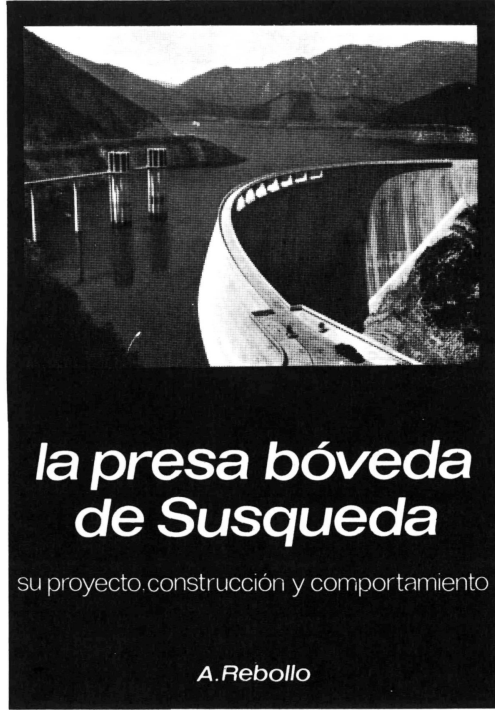

La presa bóveda de Susqueda

A. Rebollo,

Dr. Ingeniero de Caminos

El esfuerzo del constructor de presas se sitúa por su pretension de perennidad, a contracorriente de las tendencias de la civilización actual, caracte rizada por lo fungible. Pueden evocarse las 10.000 grandes. presas en funcionamiento o en construcción que están envejeciendo y reclaman los cuidado gerontológicos para mantener y perfeccionar su servicio y garantizar su inalienable pretensión de perennidad. En la medida en que todas nuevas obras, grandes o pequeñas, son portadoras de riesgos ecológicos $y$, a veces, catastróficos, que aumentan con el envejecimiento, la gerontologia de las presas es todo un emplazo. La acción adelantada de Arturo Rebollo en este terreno marca un camino a seguir para todos los que aman su propia obra con la devoción paternal que él ha puesto en Susqueda.

Un volumen encuadernado en cartoné plastificado con lomo de tela, de $18 \times 24,5 \mathrm{~cm}$, compuesto de 408 páginas, 330 figuras y fotografias y 39 tablas. 